\title{
Preparation and characterisation of photocatalytic pigments for architectural mortar based on ultramarine blue
}

\author{
Estíbaliz Aranzabe $^{1} \cdot$ Miren Blanco $^{1} \cdot$ Amaia M. Goitandia $^{1} \cdot$ Karmele Vidal $^{1} \cdot$ María Casado $^{2} \cdot$ Jose Cubillo $^{2}$
}

Received: 15 March 2019 / Accepted: 20 August 2019 / Published online: 11 September 2019

(c) The Author(s) 2019

\begin{abstract}
Architectural mortar is used in the building sector when aesthetic surface value is required and therefore, these surfaces present a great potential to be used as a solution for the reduction of atmospheric pollution. In the present work, an inorganic ultramarine blue pigment has been modified to provide the mortars with colour and photocatalytic properties, simultaneously. To modify the pigment, a sol-gel coating based on titanium n-butoxide precursor has been applied on its surface. The influence of different parameters affecting the coating formation, such as the $\mathrm{pH}$ and titanium weight content of the sol-gel reactants, has been studied. Moreover, the formation of a coating on the pigment's surface with several amounts of $\mathrm{TiO}_{2}$ anatase nanoparticles has also been explored. A pigment with better photocatalytic properties has been obtained at $\mathrm{pH}=12$ applying a coating based on titanium n-butoxide precursors (Ti precursor/pigment weight ratio $=0.5$ ) with $2 \mathrm{wt} . \%$ of titania anatase nanoparticles, maintaining the original pigment colour. Moreover, mortars with the new pigment present higher flexural strength and similar compressive strengths than non-modified mortars ensuring the applicability of the pigment in the building sector.
\end{abstract}

\section{Graphical Abstract}

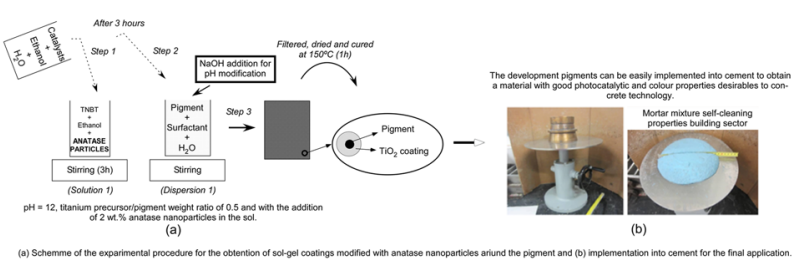

\section{Highlights}

- A photocatalytic pigment has been developed and dispersed in mortars by conventional methods to provide the mortars with colour and photocatalytic properties.

- The effect of the $\mathrm{pH}, \mathrm{Ti}$ weight content and addition of anatase nanoparticles in the coating around the pigment have been optimised during the sol-gel process.

- The photocatalytic efficiency of the obtained mortars has been analysed for its application in the building sector.

Keywords Photocatalysis $\cdot$ Self-cleaning pigment $\cdot$ Mortar $\cdot$ Anatase, Sol-gel

Estíbaliz Aranzabe

estibaliz.aranzabe@ tekniker.es

1 IK4-Tekniker, Surface Chemistry and Nanotechnology Unit, Calle Iñaki Goenaga, 5, 20600 Eibar, Gipuzkoa, Spain

2 ACCIONA Construction Technology Centre, Madrid, Spain

\section{Introduction}

The application of photocatalysis to the building sector leads to the transformation of air pollutants in harmless substances [1,2]. When air pollutants $\left(\mathrm{NO}_{x}, \mathrm{SO}_{x}\right.$, etc.) $[3,4]$ come into contact with a photocatalytic surface containing a 
semiconductor, they undergo a process of absorption, decomposition, and final transformation into limestone and mineral salts that can be easily washed away by rain. In the case of organic pollutants, the result of their complete degradation is the formation of $\mathrm{CO}_{2}$, which can be transformed into calcium carbonate, as well. The self-cleaning and pollution-reducing properties can be fundamental, especially in highly polluted areas, to increase durability and to facilitate the maintenance by making self-cleaning external surfaces [5-12].

Between the different semiconductor materials, $\mathrm{TiO}_{2}$ has gained much interest in construction materials because $\mathrm{TiO}_{2}$ is the most common among titanium minerals, which is extensively used in everyday life and is relatively inexpensive [13]. However, a considerable drawback of the use of $\mathrm{TiO}_{2}$ is the negative effect on the workability of fresh mixtures. Even if this workability can be improved by water addition, this water also affects the mechanical properties of the materials and should be controlled. Moreover, mechanical properties can be also affected upon $\mathrm{TiO}_{2}$ addition due to the nanoparticles agglomeration, which hinders the onset of the filling effect, being typical of materials prepared by adding $\mathrm{TiO}_{2}$ particles to the cement powder in a dry state [14-16].

Regarding external surfaces, in building sector it is highlighted the increasing use of coloured cement to add colour to concrete, mortar, or any other cementitious formulation, to develop exclusive, innovative surfaces, which have a degree of differentiation from their competition. For cement colouration, Ultramarine Blues are good candidates to give colour, as these pigments are resistant to the aggressiveness of strongly alkaline cement paste, and are insoluble in the mixing water and colour consistence.

Endowment of photocatalytic property on the surface of concrete structure by the only incorporation of a pigment, which can provide colour and photocatalytic properties simultaneously, but without affecting the workability of the fresh mixtures, will be a suitable solution for the development of self-cleaning coloured structures and for purification of the polluted environment [17-19]. This modified pigment will be also easily dispersed in the mortar avoiding the nanoparticle agglomeration in the final mortar.

In the present work, an inorganic ultramarine blue pigment has been modified to provide the mortars with colour and photocatalytic properties simultaneously. To modify the pigment a sol-gel coating has been applied on its surface by a previous dispersion of the pigment. Different parameters of this dispersion, affecting the subsequent coating formation around the pigment, have been optimised in order to maximise the photocatalytic effect of the pigments. Between them, there are the effects of the $\mathrm{pH}$ value, the titanium weight content of the sol-gel reactants and the addition of anatase nanoparticles. The photocatalytic efficiency of the mortar with different content of the obtained pigment have been also analysed using a Rhodamine B-based colorimetric test. Moreover, the mechanical properties of the modified mortars have been analysed to guarantee the applicability of the pigments in the building sector.

\section{Experimental procedure}

A standard ultramarine blue pigment produced by Nubiola Pigmentos S.L. was used. Ultramarine pigment compositions are based on the crystal chemistry of the royal blue Sodalite mineral Lazurite $\left[\mathrm{Na}_{6} \mathrm{Ca}_{2} \mathrm{Al}_{6} \mathrm{Si}_{6} \mathrm{O}_{24}\left(\mathrm{Sn}, \mathrm{SO}_{4}\right)_{2}\right]$.

To modify the pigment surface, the pigment was put in dispersion in water at $50 \mathrm{~g} / \mathrm{l}$ using $1 \mathrm{wt} . \%$ of PVP (polyvinylpyrrolidone) surfactant with respect to pigment weight (dispersion 1). Separately, a solution of titanium (IV) $n$-butoxide precursor (TNBT)) was mixed with ethanol and a mixture of hydrochloric acid, water and ethanol was added drop by drop to the ethanol/titanium mixture (molar ratio: TNBT/ethanol/ $\mathrm{H}_{2} \mathrm{O} / \mathrm{HCl}$ of $1 / 50 / 2 / 0.04$ ) (solution 1). The resulting solution were kept under stirring during $3 \mathrm{~h}$. After this time, the required weight of solution 1 was added drop by drop to dispersion 1 (Fig. 1) with the help of an automatic dispenser during $1 \mathrm{~h}$. Pigments with different Ti/pigment weight ratios were prepared following this process. After the corresponding solutions were added,
Fig. 1 Scheme of the experimental procedure for the obtention of the sol-gel coatings around the pigment

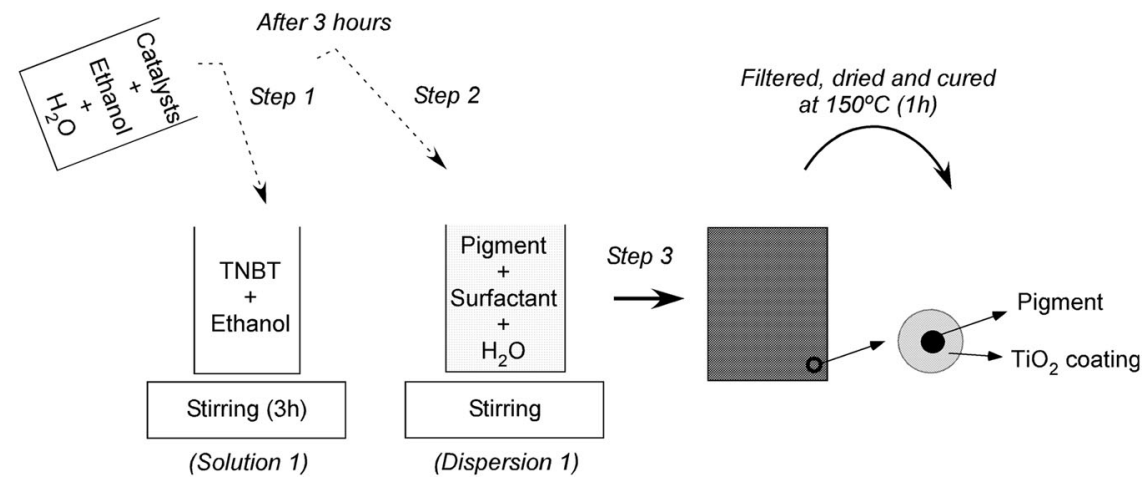


Fig. 2 Experimental test chamber

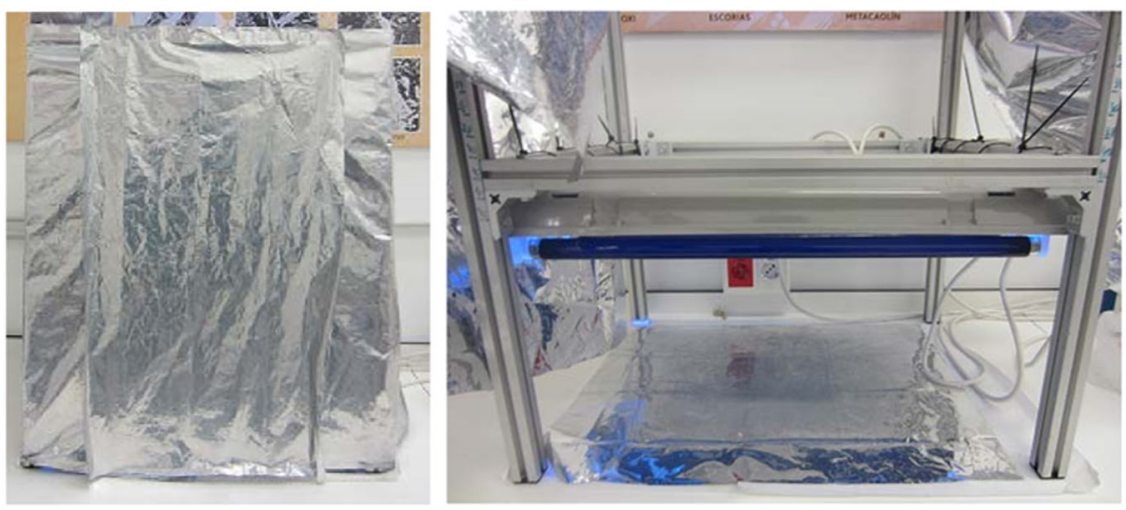

the mixture with a $\mathrm{pH}$ of 10 was maintained under stirring during $3 \mathrm{~h}$ and filtered, and finally cured at $150{ }^{\circ} \mathrm{C}$ for $1 \mathrm{~h}$. This temperature has been selected far enough from the degradation temperature of the pigment indicated by the pigment manufacturer $\left(350^{\circ} \mathrm{C}\right)$ in order to avoid whitening of the pigment and losing of colour due to a degradation of the chromophore group of the pigment.

This temperature is considered the maximum possible value of curing temperature for the coatings, as the chromophore group of the pigment degrades at higher temperatures.

Titanium concentration of pigments was measured by X-ray fluorescence with a Bruker S8 Tiger under vacuum or helium gas depending on their nature. The software Spectra Plus was employed to deconvolute and integrate the elemental profiles to get the quantification of chemical elements.

Laser Dispersion was employed to obtain the particle size distribution $(0.02-2000 \mu \mathrm{m}$ range) of the samples by using a Malvern-Mastersizer 2000. Water was used as the dispersion media and a particle refractive index of 1.510 was considered for the measurement and five cycles of measurement were done.

For transmission electron microscopy (TEM) measurements, the powder samples were deposited on a grid covered with a holey carbon film and transferred to Hitachi HT7700 electron microscope operating at $80 \mathrm{kV}$.

To evaluate the photocatalytic efficiency of the pigments, two different alternatives were studied: (a) to press the pigment powder to prepare a pigment pill with a press machine and (b) to include the pigment in the mortar (the final matrix) by mixing $450 \mathrm{~g}$ of cement with $225 \mathrm{~g}$ of water, $1350 \mathrm{~g}$ of sand and different quantities of pigment according to the Standard UNE 196-1 [20].

The photocatalytic efficiency was measured using a Rhodamine B (N, N, N', N'-Tetraethylrhodamine chloride)based colourimetric test. Cement pastes substrates were prepared by mixing $500 \mathrm{~g}$ of cement with $150 \mathrm{~g}$ of water according to the Standard UNE-EN 196.3. The coating was applied on the cement paste substrate by rolling. The Rhodamine B dye exhibits a strong red colour due to its highly delocalised П-electron system. It is very soluble in water and can therefore be spread uniformly on a given surface. Rhodamine B was spread on the pigment pull or the pigment modified, and consequently, its discolouration can be followed by colourimetry.

The specimens were placed horizontally with an UVA light source located above the surface of the specimens (see Fig. 2). The light intensity received by the specimens was $10 \mathrm{~W} / \mathrm{m}^{2}$. The discolouration of the rhodamine was followed by colourimetry.

The results are expressed in the CIELAB system with $L^{*}$, $a^{*}$ and $b^{*}$ colourimetric coordinates. As rhodamine $\mathrm{B}$ applied onto materials gave an initial pink colouration, only the colourimetric coordinate $a^{*}$ was considered. The measurements of $a^{*}$ were performed at regular time intervals during $9 \mathrm{~h}$. To quantify the photocatalytic activity, an index of the photocatalytic activity (PAI) was determined: $\mathrm{PAI}=$ $C-$ Co [20] where: $C$ is the activity coefficient of the photocatalytic specimen and $\mathrm{Co}$ is the activity coefficient of the reference specimen. The activity coefficient $(C)$ could be defined as: $C=\left[\operatorname{At}\left(a^{*}\right)-A\left(a^{*}\right)\right] / \operatorname{At}\left(a^{*}\right)$. For this work, a value of PAI $>0.1$ after $9 \mathrm{~h}$ has been established as an acceptable value when mortar with the dispersed pigment is evaluated.

Energy-dispersive X-ray spectroscopy (EDX) and mapping analysis using a scanning electron microscope Hitachi S-4800 was carried out to evaluate the dispersion of the developed Pigments in mortar.

The flexural and compressive strength of hardened mortar was determined at 3, 7 and 28 days following the Standard UNE-EN 196.1. The flexural strength was determined by three-point loading of a prism specimen. Subsequenly to the failure and breakage of this specimen, the compressive strength was determined on each half of the prism. The dimensions of the specimens were $160 \times 40 \times 40 \mathrm{~mm}$.

The Brunauer-Emmett-Teller (BET)-specific surface area was measured by low-temperature nitrogen adsorption/ 
desorption isotherms using a Quantachrome Autosorb iQ analyser. Typically, 0.1-0.2 g of a sample was used for the measurement. Prior to nitrogen adsorption at $77 \mathrm{~K}$, the sample was degassed at $573 \mathrm{~K}$.

\section{Results and discussion}

In order to optimise the development of an inorganic ultramarine blue pigment capable of providing colour and photocatalytic properties simultaneously to the cement, we have modified the conventional pigment into a photocatalytic one coating the pigment with an inorganic sol-gel coating. The effect of the Titanium weight content, $\mathrm{pH}$ value and the addition of anatase nanoparticles in the pigment coating on the self-cleaning properties of the pigment has been analysed.

\subsection{Effect of titanium weight content on the pigment coating in the self-cleaning properties}

In the pigment preparation process, different weight ratios of Ti precursors/pigment were employed. After filtering and curing, pigments with different Ti content $(0.10,0.25$ and 0.50 ), on their surfaces and therefore, with different properties have been obtained. In Table 1, there are listed the titanium weight contents present in the pigment measured by X-ray fluorescence, the results of maximum index of photocatalytic activity (PAI) and mean particle size $\left(d_{(0.5)}\right)$ values obtained for each pigment sample. Moreover, Table 1 shows the evolution of PAI values with the time.
As it is expected, increasing the Ti precursor weight with respect to the pigment weight, there are obtained pigments with higher Ti contents. However, the sample named as S3_10 (having a titanium content of $1.42 \mathrm{wt} . \%$ ) reaches the same PAI value at $9 \mathrm{~h}$ than the pigment S1_10 (having a titanium content of $7.25 \mathrm{wt} . \%$ ).

The reason for this behaviour could be the pigment agglomeration (Table 1). The sample obtained from S3_10 is an easily dispersible pigment with a mean particle size of $0.94 \mu \mathrm{m}$, while the sample obtained from S1_10 has a mean particle size of $9.95 \mu \mathrm{m}$. As it is known, the active surface of the agglomerated sample is lower than the one of the nonagglomerated sample, being more difficult to obtain a good contact between the organic molecule (Rhodamine B) and the titanium, and then, the sample shows lower value of PAI [21-23].

The pigment providing the highest PAI value and the lowest agglomeration (S3_10), was selected to study the self-cleaning capability of the pigment modified mortar. The concrete matrix was prepared using a concentration of $7 \mathrm{wt} . \%$ pigment with respect to cement.

Figure 3 shows an EDX Ti mapping of a non-modified and modified mortar with pigment (S3_10). As it can be observed, this amount of $\mathrm{Ti}$ is homogeneously distributed inside the mortar.

However, in spite of the good Ti distribution the PAI value of the mortar is smaller than 0.1 . So, it was decided to study different alternatives to obtain self-cleaning pigments. For this, the titanium concentration (wt.\%) is increased by using two different strategies: varying the $\mathrm{pH}$ and adding anatase nanoparticles in the sol, having fixed the weight
Table 1 Titanium weight content (wt.\%) in the pigments, mean participle size values and maximum PAI values obtained for the samples

Fig. 3 EDX Ti mapping of the mortars with 7 wt. \% of a nonmodified and $\mathbf{b}$ modified pigment

\begin{tabular}{lllll}
\hline Samples & $\begin{array}{l}\text { Weight ratio Ti } \\
\text { precursor/pigment }\end{array}$ & $\begin{array}{l}\text { Measured titanium weight content } \\
(\text { wt.\% }) \text { on the pigment }\end{array}$ & $\begin{array}{l}d_{(0.5)} \\
(\mu \mathrm{m})\end{array}$ & $\begin{array}{l}\text { Maximum PAI value } \\
(9 \mathrm{~h})\end{array}$ \\
\hline S1_10 & 0.50 & 7.25 & 9.95 & 0.116 \\
S2_10 & 0.25 & 3.50 & 8.52 & 0.072 \\
S3_10 & 0.10 & 1.42 & 0.94 & 0.115 \\
\hline
\end{tabular}
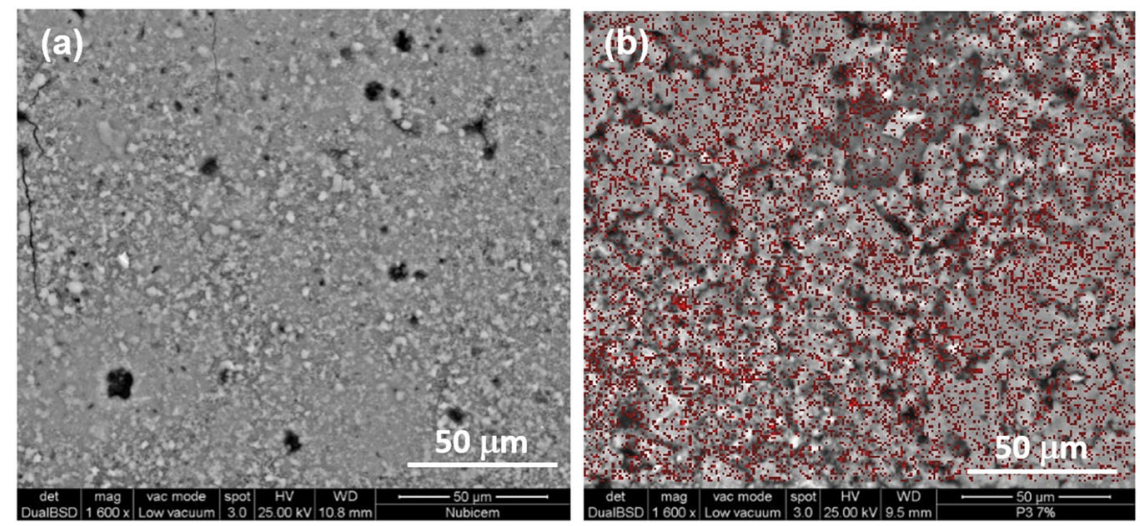
ratio Ti precursor /pigment, in both cases, to 0.50 (S1) and $0.25(\mathrm{~S} 2)$.

\subsection{Effect of the pH value in the pigment coating formation in the photocatalytic efficiency}

In the general methods for obtaining the coated pigments, after adding the $\mathrm{Ti}$ precursor solution in the pigment

Table 2 Titanium weight content (\%) in the pigments, mean participle size values and maximum PAI values for obtained pigments

\begin{tabular}{|c|c|c|c|c|c|}
\hline Samples & $\mathrm{pH}$ & $\begin{array}{l}\text { Weight ratio Ti } \\
\text { precursor } \\
\text { /pigment }\end{array}$ & $\begin{array}{l}d_{(0.5)} \\
(\mu \mathrm{m})\end{array}$ & $\begin{array}{l}\text { Ti weight } \\
\text { content }(\%) \text { on } \\
\text { the pigment }\end{array}$ & $\begin{array}{l}\text { Maximum } \\
\text { PAI value } \\
(9 \mathrm{~h})\end{array}$ \\
\hline $\mathrm{S} 1 \_10$ & 10 & & 9.92 & & 0.116 \\
\hline $\mathrm{S} 1 \_11$ & 11 & & 8.38 & & \\
\hline $\mathrm{S} 1 \_12$ & 12 & 0.50 & 6.01 & 7.25 & 0.143 \\
\hline $\mathrm{S} 1 \_13$ & 13 & & 19.51 & & \\
\hline S2_10 & 10 & & 8.52 & & 0.072 \\
\hline S2_11 & 11 & & 5.88 & & \\
\hline S2_12 & 12 & 0.25 & 3.72 & 3.50 & 0.170 \\
\hline S2_13 & 13 & & 10.78 & & \\
\hline
\end{tabular}

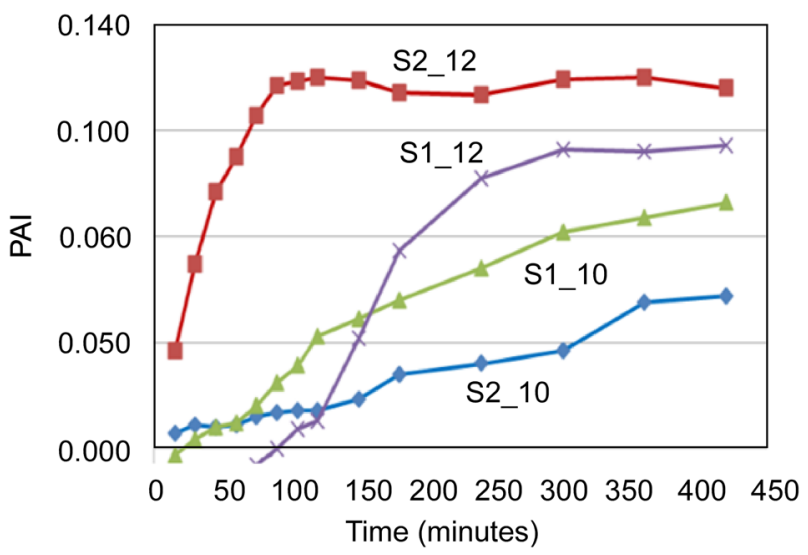

Fig. 4 Evolution of PAI with time of S1_10, S1_12, S2_10 and S2_12 pigments dispersion, the mixture was maintained under starring at $\mathrm{pH}=10$ for $3 \mathrm{~h}$. It is well known that the $\mathrm{pH}$ parameter affects the sol-gel hydrolysis and condensation reaction rates [24, 25], and therefore, the $\mathrm{pH}$ could affect the agglomeration of the pigment during the coating formation. In this work, the effect of four values of $\mathrm{pH}(\mathrm{pH}=10,11$, 12 and 13) has been studied. For this, $\mathrm{NaOH}$ was added to increase the initial $\mathrm{pH}$ value $(\mathrm{pH}=10)$. The values of mean participle size obtained for each experiment are shown in Table 2 .

Less agglomerated pigments (S1_12 and S2_12) were achieved using $\mathrm{pH}=12$ and they show better properties in terms of self-cleaning ability, compared with S1_10 and S2_10).

Figure 4 shows the evolution of PAI values with time for each sample obtained at $\mathrm{pH}=12$. The maximum PAI value obtained for the S1_10, S1_12, S2_10 and S2_12 samples have been $0.116,0.143,0.072$ and 0.170 , respectively.

As can be seen, pigment obtained using the conditions of S2_12 presents the highest PAI values, even if it is not the pigment with the highest titanium concentration. This behaviour could be due to the better dispersibility of this pigment $\left(d_{(0.5)}=3.72 \mu \mathrm{m}\right)$ with respect to the others.

Taking into account the obtained results, the developed pigments providing the highest PAI values were dispersed in the concrete matrix (the mortar was prepared as explained before). However, S1_12 and S2_12 developed pigments do not have enough self-cleaning ability when they are dispersed in concrete.

\subsection{Effect of the addition of anatase nanoparticles on the pigment coating in the self-cleaning properties}

Another via to increase the titanium concentration is the addition of anatase nanoparticles in the coating deposited around the pigment surface $[16,26]$. Different concentrations of $\mathrm{TiO}_{2}$ nanoparticles were added to the titanium (IV) $n$-butoxide solution and then, it was hydrolysed and added drop by drop to the pigment dispersion (Fig. 5). For this
Fig. 5 Scheme of the experimental procedure for the obtention of sol-gel coatings modified with anatase nanoparticles around the pigment

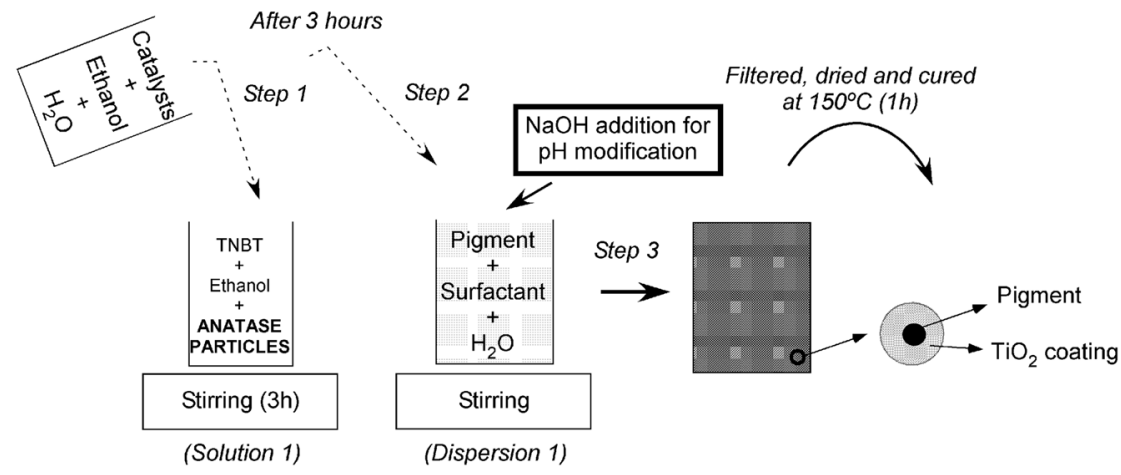


Table 3 Titanium weight content (wt.\%) in the pigments, mean particle size values and DL results for the pigments

\begin{tabular}{llllll}
\hline Samples & $\begin{array}{l}\text { Weight ratio Ti precursor } \\
\text { /pigment }\end{array}$ & $\begin{array}{l}\text { Ti wt.\% } \\
\text { particles }\end{array}$ & $\begin{array}{l}\text { Measured Ti wt. on } \\
\text { the pigment }\end{array}$ & $\begin{array}{l}d_{(0.5)} \\
(\mu \mathrm{m})\end{array}$ & DL \\
\hline S1_12_anatase & 0.50 & 2.0 & 14.00 & 6.0 & 2.0 \\
S2_12_anatase & 0.25 & 5.0 & 10.80 & 3.8 & 3.0 \\
\hline
\end{tabular}

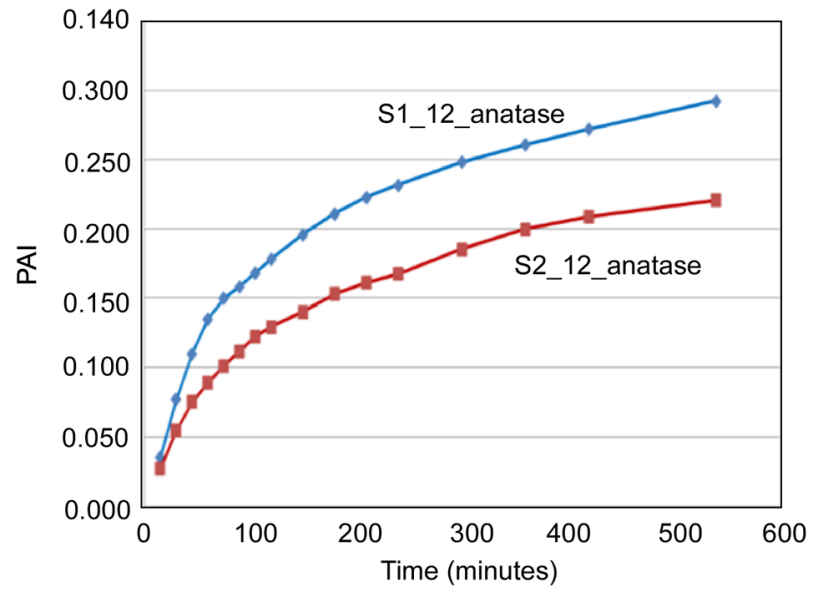

study, it has been decided to work under $\mathrm{pH}=12$ considering previous results that demonstrate that the use of $\mathrm{pH}=12$ reduce the agglomeration.

The titanium precursor/pigment weigh ratios employed in this analysis are 0.5 and 0.25 (see Table 3). As it was expected, the pigments obtained in the process employing higher titanium precursor/pigment weight ratios present higher Ti wt.\%. For the samples S1_12 and S2_12 with anatase particles (now S1_12_anatase and S2_12_anatase) the measured titanium weight content (wt.\%) on the pigment by XRF is 14.00 (before, it was 7.25) and 10.80 (before, it was 3.50), respectively.

In addition, particle size distribution, $\mathrm{d}_{50}$, is 6 and 3.80 for S1_12_anatase and S2_12_anatase samples, respectively. So, a bigger agglomeration is observed in the pigments obtained with higher Ti:pigment ratio (S1_12_anatase).

Figure 6 shows the PAI evolution with time for both samples. The maximum PAI value obtained for S1_12_anatase and S2_12_anatase is 0.293 and 0.221 , respectively.

The obtained results indicate that both pigments provided high PAI values. Therefore, both pigments were dispersed in the concrete matrix using a concentration of $7 \mathrm{wt} . \%$ pigment with respect to cement obtaining maximum PAI values (9h) (see Fig. 7) of 0.304 for the pigment S1_12_anatase and 0.227 for the pigment S2_12_anatase, respectively. So, both of the obtained pigments can be considered as self-cleaning pigments, because both can provide the mortar where they are included with a PAI values after $9 \mathrm{~h}$, higher than the established as minimum value (PAI >0.1).

The PAI values of the modified mortars are higher than the expected ones considering the amount of pigment inside. This can be explained by the higher roughness on mortars than in pigments, leading to a higher surface contact area between the Rhodamine B molecule and the Ti on the mortars [27].

In order to select the best composition, the change in DL (lightness of the colour) of both pigments with respect to the original pigment has been measured, obtaining a value of 2 and 3 for the sample S1_12_anatase and S2_12_anatase, respectively.

Consequently, taking into account these last results, the sample S1_12_anatase has been selected as the pigment with the optimal properties due to its better self-cleaning properties and lowest colour change. Moreover, TEM image of the S1_12_anatase pigment (Fig. 8) does not differ significantly from the image of raw material (non-modified
Fig. 6 Evolution of PAI with time for S1_12_anatase and S2_12_anatase pigments

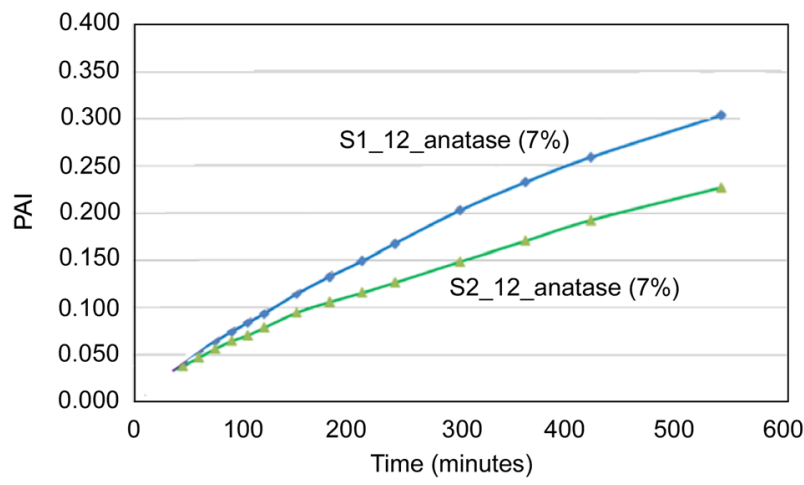

Fig. 7 Evolution of PAI with the time of pigment S1_12_anatase (7 wt.\%) and S2_12_anatase (7 wt.\%) dispersed in concrete

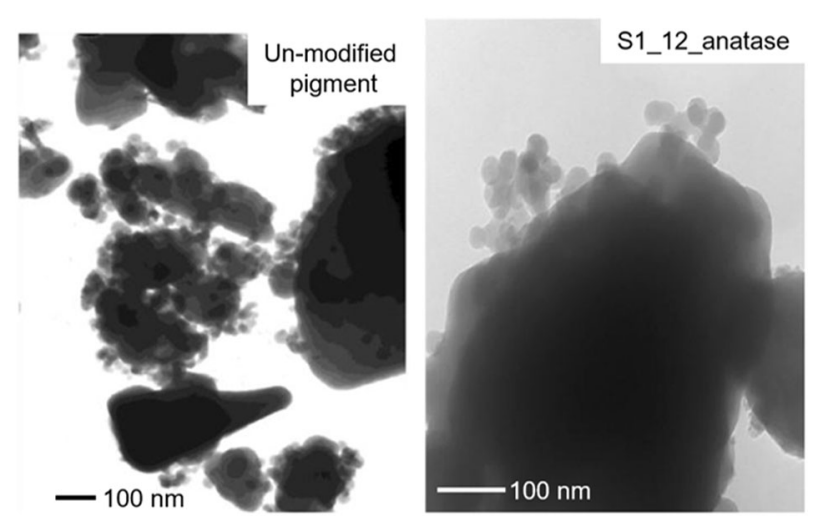

Fig. 8 TEM micrographs for non-modified and S1_12_anatase pigments 
Table 4 Mechanical properties of the prepared mortar specimens obtained by at least three specimens

\begin{tabular}{llllllll}
\hline $\begin{array}{l}\text { Days } \\
\text { Samples }\end{array}$ & 3 & 7 & 28 & 3 & 7 & 28 \\
\hline 0 wt.\% pigment & Flexural & strength & (MPa) & & \multicolumn{2}{l}{ Compressive strength (MPa) } \\
7 wt.\% non-modified pigment & $2.8(1)$ & $4.5(1)$ & $5.7(1)$ & $21.2(2)$ & $31.5(2)$ & $31.4(2)$ \\
7 wt.\% modified S1_12_anatase pigment & $3.1(1)$ & $4.5(1)$ & $4.9(1)$ & $25.2(1)$ & $27.7(2)$ & $28.9(2)$ \\
& $3.6(1)$ & $4.8(1)$ & $8.2(1)$ & $23.7(2)$ & $26.1(1)$ & $30.2(2)$ \\
\hline
\end{tabular}

pigment). Some particles are observed on both pigments, as the original ultramarine blue employed in this study has a precursor silica-based coating to improve its resistance to acidic medium.

To ensure the applicability of the pigments in the building sector, the mechanical properties of mortars modified with a $7 \mathrm{wt} . \%$ of pigment have been measured.

Table 4 collects the results of the mechanical properties of the mortar specimens. Each of the presented data was obtained by at least three specimens in order to get accuracy and to avoid probable errors.

From the results, it can be seen that the addition of the modified pigment improves the flexural strength of the reference mortar (without pigment) and of mortar with the non-modified pigment. The compressive strength of the mortar with non-modified and modified pigment are very similar to the reference mortar [28, 29]. This can be due to the higher specific area of the modified pigment $\left(61 \mathrm{~m}^{2} / \mathrm{g}\right)$ with respect to non-modified one $\left(14 \mathrm{~m}^{2} / \mathrm{g}\right)$. This gives rise to a higher interaction pigment mortar and to an improvement of the mechanical properties.

\section{Conclusions}

The increasing use of coloured architectural mortars can be used as a solution to the high atmospheric pollutant concentration, the source of which often is related to infrastructure construction. In this study, a photocatalytic ultramarine blue pigment has been developed by modifying its surface with a sol-gel coating. To increase the photocatalytic activity of the pigment towards the degradation of Rhodamine B different alternatives were studied: increasing the titanium weight content of the pigment by increasing the titanium precursor amount in the modification process, modifying the $\mathrm{pH}$ value employed in the pigment modification process and to add $\mathrm{TiO}_{2}$ anatase nanoparticles during the modification process to obtain an ultramarine blue pigment coated with $\mathrm{TiO}_{2}$ films containing different concentrations of nanoparticles. The developed pigments can be easily implemented into cement to obtain a material with good photocatalytic and colour properties desirables to concrete technology. The experimental results obtained after the mixture of the pigment with the cement have shown that the better self-cleaning properties and lowest colour change have been achieved by using a pigment obtained under $\mathrm{pH}=12$ and employing a titanium precursor/pigment weight ratio of 0.5 and with the addition of 2 wt.\% anatase nanoparticles in the sol. Moreover, mortars with the new pigment present higher flexural strength and similar compressive strengths than non-modified mortars. Ensuring the applicability of the pigment in the building sector.

\section{Disclaimer}

This manuscript has not been submitted to, nor is under review at, another journal or other publishing venue.

Acknowledgements This project has received funding from the European Union Seventh Framework Programme (FP7-NMP-2010-Small5) under grant agreement no. 280393 and the Basque Government within the Elkartek Programme, ACTIMAT 2018-2019 (KK-2018/ 00099).

Author contributions All authors have participated in (a) conception and design, or analysis and interpretation of the data; (b) drafting the article or revising it critically for important intellectual content; and (c) approval of the final version.

\section{Compliance with ethical standards}

Conflict of interest The following authors have no affiliations with organisations with direct or indirect financial interest in the subject matter discussed in the manuscript: EA, MB, AMG, KV are affiliated to IK4-Tekniker, Surface Chemistry and Nanotechnology Unit; MC, $\mathrm{JC}$ are affiliated to ACCIONA Construction Technology Centre.

Publisher's note Springer Nature remains neutral with regard to jurisdictional claims in published maps and institutional affiliations.

Open Access This article is distributed under the terms of the Creative Commons Attribution 4.0 International License (http://crea tivecommons.org/licenses/by/4.0/), which permits unrestricted use, distribution, and reproduction in any medium, provided you give appropriate credit to the original author(s) and the source, provide a link to the Creative Commons license, and indicate if changes were made.

\section{References}

1. Cohen JD, Sierra-Gallego G, Tobón JI (2015) Evaluation of photocatalytic properties of portland cement blended with titanium oxynitride $\left(\mathrm{TiO}_{2-\mathrm{x}} \mathrm{N}_{\mathrm{y}}\right)$ nanoparticles. Coatings 5:465-476 
2. Sharma VP, Sharma U, Chattopadhyay M, Shukla VN (2018) Advance applications of nanomaterials: A review. Mater Today: Proc 5:6376-6380

3. Kim DJ, Nasonova A, Park JH, Kang JY, Kim KS (2007) $\mathrm{NO}_{x}$ and $\mathrm{SO}_{x}$ removal by low temperature plasma-photocatalysts hybrid system. Mater Sci Forum 544-545:91-94

4. Mills A, Elouali S (2015) The nitric oxide ISO photocatalytic reactor system: Measurement of NOx removal activity and capacity. J Photoch Photobio A 305:29-36

5. Enea D, Luca Guerrini G (2010) Photocatalytic properties of cement-based plasters and paints containing mineral pigments. Transportation Research Record. J Transp Res Board 214:52-60

6. Cárdenas C, Tobón JI, García C, Vila J (2012) Functionalized building materials: photocatalytic abatement of $\mathrm{NO}_{\mathrm{x}}$ by cement pastes blended with $\mathrm{TiO}_{2}$ nanoparticles. Constr Build Mater 36 (1):820-825

7. Teleki A, Wengeler R, Wengelera L, Nirschl H, Pratsinis SE (2008) Distinguishing between aggregates and agglomerates of flame-made $\mathrm{TiO}_{2}$ by high-pressure dispersion. Powder Technol 181:292-300

8. Lucas SS (2017) Influence of operating parameters and ion doping on the photocatalytic activity of mortars containing titanium dioxide nanoparticles. Mater Today: Proc 4:11588-11593

9. Zhong L, Haghighat F, Blondeau P, Kozinski J (2010) Modeling and physical interpretation of photocatalytic oxidation efficiency in indoor air applications. Build Environ 45(12):2689-2697

10. Lucas SS, Ferreira VM, Aguiar JLBD (2013) Incorporation of titanium dioxide nanoparticles in mortars - Influence of microstructure in the hardened state properties and photocatalytic activity. Cement. Concr Res 43:112-120

11. Sikora P, Horszczaruk E, Rucinska T (2015) The effect of nanosilica and titanium dioxide on the mechanical and selfcleaning properties of waste-glass cement mortar. Procedia Eng 108:146-153

12. Verbruggen CSW, Tytgat T, Van Passel S, Martens JA, Lenaerts $\mathrm{S}$ (2014) Cost-effectiveness analysis to assess commercial $\mathrm{TiO}_{2}$ photocatalysts for acetaldehyde degradation in air. Chem Pap 68 (9): 1273-1278

13. Diamanti MV, Pedeferri MP (2019) Nanotechnology in ecoefficient construction. Materials processes and applications. Photocatalytic performance of mortars with nanoparticles exposed to the urban environment. In: Pacheco-Torgal F, Diamanti MV, Nazari A, Granqvist CG, Pruna A, Amirkhanian S (eds), Woodhead Publishing Series in Civil and Structural Engineering, Nanotechnology in Eco-efficient Construction. 2nd edn. 527-555

14. Pérez-Nicollas M, Navarro-Blasco Í, Fernández JM, Alvarez JI (2017) The effect of $\mathrm{TiO}_{2}$ doped photocatalytic nano-additives on the hydration and microstructure of portland and high alumina cements. Nanomaterials 7(10):329(1)-329(21)

15. Rashad AM (2015) A synopsis about the effect of nano-titanium dioxide on some properties of cementitious materials e a short guide for civil engineer. Adv Mater Sci 40:72-88
16. Diamanti MV, Lollini F, Pedeferri MP, Bertolini L (2013) Mutual interactions between carbonation and titanium dioxide photoactivity in concrete. Build Environ 62:174-181

17. Hedayati HR, Sabbagh Alvani AA, Sameie H, Salimi R, Moosakhani S, Tabatabaee F, Amiri Zarandi A (2015) Synthesis and characterization of $\mathrm{Co}_{1-\mathrm{x}} \mathrm{Zn}_{\mathrm{x}} \mathrm{Cr}_{2-\mathrm{y}} \mathrm{Al}_{\mathrm{y}} \mathrm{O}_{4}$ as a near-infrared reflective color tunable nano-pigment. Dyes Pigments 113:588-595

18. Han A, Ye M, Liu L, Feng W, Zhao M (2014) Estimating thermal performance of cool coatings colored with high near-infrared reflective inorganic pigments: Iron doped $\mathrm{La}_{2} \mathrm{Mo}_{2} \mathrm{O}_{7}$ compounds. Energ Build 84:698-703

19. Aranzabe E, Villasante PM, March R, Arriortua MI, Vadillo J, Larrañaga A, Aranzabe A (2016) Preparation and characterization of high NIR reflective pigments based in ultramarine blue. Energ Build 126:170-176

20. Ruot B, Plassais A, Olive F, Guillot L, Bonafous L (2009) $\mathrm{TiO}_{2^{-}}$ containing cement pastes and mortars: measurements of the photocatalytic efficiency using a rhodamine B-based colourimetric test. Sol Energy 83:1794-1801

21. Ricci PC, Carbonaro CM, Lehmann AG, Congiu F, Puxeddu B, Cappelletti G, Spadavecchi F (2013) Structure and photoluminescence of $\mathrm{TiO}_{2}$ nanocrystals doped and co-doped with $\mathrm{N}$ and rare earths $\left(\mathrm{Y}^{3+}, \mathrm{Pr}^{3+}\right)$. J Alloy Compd 561:109-113

22. Wanga H, Huang S-1, Zuo Y-j, Zhou T-r, Zhang L-r (2011) Corrosion resistance of lamellar aluminium pigments coated by $\mathrm{SiO}_{2}$ by sol-gel method. Corros Sci 53:161-167

23. Zhang D, Zhao Y, Chen L (2018) Fabrication and characterization of amino-grafted graphene oxide modified $\mathrm{ZnO}$ with high photocatalytic activity. Appl Surf Sci 458:638-647

24. Lettieri M, Colangiuli D, Masieri M, Calia A (2019) Field performances of nanosized $\mathrm{TiO}_{2}$ coated limestone for a self-cleaning building surface in an urban environment. Build Environ 147:506-516

25. Alias SS, Ismail AB, Mohamad AA (2010) Effect of pH on $\mathrm{ZnO}$ nanoparticle properties synthesized by sol-gel centrifugation. $\mathrm{J}$ Alloy Compd 499:231-237

26. Zhang W, Chen S, Yui S, Yin Y (2007) Experimental and theoretical investigation of the $\mathrm{pH}$ effect on the titania phase transformation during the sol-gel process. J Cryst Growth 308:122-129

27. El-Sherbiny S, Morsy F, Samir M, Fouad OA (2014) Synthesis, characterization and application of $\mathrm{TiO}_{2}$ nanopowders as special paper coating pigment. Appl Nanosci 4:305-313

28. Just da Costa e Silva A, Carasek H, de Freitas Mota JM, Rodrigues F (2011) An experimental study on how treated bases influence the adhesion of mortar coatings. XII DBMC: 12th International Conference on Durability of Building Materials and Components. Porto (Portugal, 12th-15th April).

29. Reis JML, Moreira DC, Nunes LCS, Sphaier LA (2011) Experimental investigation of the mechanical properties of polymer mortars with nanoparticles. Mat Sci Eng: A 528 (18):6083-6085 